\title{
Erwerbsverläufe beim Übergang in den Ruhestand
}

\author{
Tanja Zähle \\ Katja Möhring \\ Peter Krause
}

Die gegenwärtige Debatte um die Erwerbsbeteiligung von Menschen ab 55 Jahren fokussiert vor allem auf die Themen Arbeitslosigkeit, Frühverrentung und Rente mit 67. Der Ausstieg aus dem Erwerbsleben wird dabei als Zäsur im Lebensverlauf aufgefasst. Tatsächlich erfolgt der Übergang in den Ruhestand zumeist stufenweise, eingeleitet durch eine schrittweise Reduktion der Arbeitszeit oder in den meisten Fällen - durch Arbeitslosigkeit. Diese Erwerbsmuster beim Übergang in den Ruhestand prägen nicht nur das aktuelle Einkommen, sondern auch das zukünftige Renteneinkommen. Je prekärer sich daher der Übergangsprozess gestaltet, desto problematischer ist der Ausblick auf das spätere Alterseinkommen.

\section{Einleitung}

Die Zielsetzung der deutschen Renten- und Sozialpolitik zur Flankierung des Übergangs in den Ruhestand hat in den letzten 30 Jahren mehrfache Wandlungen durchlaufen. Nach der Forcierung einer möglichst frühen Verabschiedung aus dem Erwerbsleben in den 1970er Jahren, erfolgte in den letzten Jahren eine Kehrtwendung hin zu einem längeren Verbleib auf dem Arbeitsmarkt. Inwieweit das politische Ziel, die Erwerbsbeteiligung der Bevölkerung ab 55 Jahren zu erhöhen, erfolgreich umgesetzt werden kann, ist allerdings umstritten (Bäcker 2006). Überdurchschnittlich hohe Arbeitslosigkeit sowie eine Erwerbsquote von knapp $50 \%$ deuten derzeit auf eher geringe Chancen von Älteren auf dem Arbeitsmarkt hin (BMFSFJ 2005). Zudem nimmt ab 55 mit jedem weiteren Altersjahr die Arbeitsmarktpartizipation ab, sodass von den 64-Jährigen weniger als $20 \%$ noch erwerbstätig sind (Puch 2009).

Der eigentliche Übergang in den Ruhestand, also der Wechsel aus Erwerbsarbeit (oder Nichterwerbstätigkeit) in die Rente, ist um ein vielfaches komplexer als dies auf den ersten Blick erscheint, denn in den seltensten Fällen vollzieht sich der Übergang von einem Tag auf den anderen. Der Renteneinstieg ist vielmehr ein Prozess innerhalb des individuellen Lebensverlaufs, der häufig bereits Jahre vor Erreichen des Rentenalters mit einer schrittweisen Reduzierung der Erwerbsarbeit eingeleitet wird und erst stufenweise zur völligen Aufgabe der Erwerbstätigkeit führt (Voges 2008). Dementsprechend verbergen sich hinter den aggregierten Zahlen zur Erwerbsbetei- ligung Älterer im individuellen Verlauf vielfältige Erwerbssequenzen bis zum Eintritt in den Ruhestand.

Vor diesem Hintergrund werden im vorliegenden Beitrag die Erwerbsverläufe im Übergang in den Ruhestand anhand der innovativen Methode der Sequenzanalyse beschrieben. Diese Methode erlaubt es, die Vielfalt individueller Verlaufspassagen sichtbar zu machen und diese zu Verlaufstypen zusammenzufassen. Dadurch wird deutlich, wie verbreitet bestimmte Formen des Übergangs aus dem Erwerbsleben in die Rente - z.B. direkte Einstiege aus (Vollzeit-)Erwerbstätigkeit oder gleitende Übergänge aus anderen Formen der Erwerbsbeteiligung, aus Arbeitslosigkeit und Nicht-Erwerbstätigkeit - sind. Im folgenden Analyseschritt werden die mit den Erwerbsverläufen korrespondierenden Einkommensverläufe betrachtet, um die enge Verbindung von Erwerbsübergängen und Einkommen und deren sozialpolitische Bedeutung hervorzuheben. Die Auswertungen konzentrieren sich auf die zehn Lebensjahre vor dem gesetzlichen Renteneintrittsalteralter - also auf die Altersspanne 55 bis 65 Jahre. Analysegruppe sind Ostund Westdeutsche sowie Männer und Frauen der Geburtsjahrgänge 1937 bis 1941, der Analysezeitraum erstreckt sich von 1992 bis 2006.

Die sozialpolitischen Regelungen, die den Übergang in den Ruhestand flankieren, behandelt Abschnitt 2. Abschnitt 3 skizziert das Erwerbsverhalten Älterer vor dem Renteneinstieg. Die anschließenden empirischen Analysen (Abschnitt 4) werden eingeleitet erstens mit einem kurzen Hinweis auf die Datenbasis (Sozio-oekonomisches Panel (SOEP)), zweitens der Abgrenzung der zentralen Indikatorvariable, dem Erwerbsstatus, sowie drittens ei- ner kurzen, anwendungsorientierten Einführung in die Methode der Sequenzmusteranalyse (4.1). Daran anknüpfend erfolgt die empirische Quantifizierung der Erwerbsverläufe der 55-65-Jährigen auf Basis der Sequenzmusteranalyse (4.2) sowie eine Gruppierung der so ermittelten Verlaufsmuster anhand einer Clusteranalyse (4.3); die sich hieraus ergebenden sechs unterschiedlichen Verlaufstypen werden dann sozio-demografisch nach Ost-West- sowie Geschlechtsunterschieden weiter differenziert (4.4). Anhand der Betrachtung der individuellen und haushaltsbezogenen Einkommensentwicklung werden schließlich noch die Auswirkungen der unterschiedli-

Tanja Zähle, Dipl.-Soz., ist wissenschaftliche Referentin am Statistischen Landesamt Baden-Württemberg. Von 2005-2009 war sie wissenschaftliche Mitarbeiterin am Institut für Soziologie der Ludwig-MaximilianUniversität München e-mail: tanja.zaehle@stala.bwl.de Katja Möhring, Dipl.-Sozia/wiss., ist Promotions-Stipendiatin im Graduiertenkolleg SOCLIFE an der Universität zu Köln. Von 2006-2009 war sie studentische Mitarbeiterin der Längsschnittstudie Soziooekonomisches Panel (SOEP) am Deutschen Institut für Wirtschaftsforschung (DIW) Berlin.

e-mail moehring@wiso.uni-koeln.de Peter Krause, Dr., ist wissenschaftlicher Mitarbeiter der Längsschnittstudie Sozio-oekonomisches Panel (SOEP) am DIW Berlin. Arbeitsschwerpunkte: Einkommen, Verteilung, Armut und Dynamik; objektive Lebenslagen, wahrgenommene Lebensqualität, capabilities, soziale Indikatoren. e-mail: pkrause@diw.de 
chen Erwerbsverläufe auf das spätere Renteneinkommen erfasst (4.5). Der Beitrag schließt mit einer kurzen Zusammenfassung und einer offenen sozialpolitischen Deutung der empirischen Befunde (Abschnitt 5).



Eingeleitet wurde die Politik der möglichst frühen Freisetzung älterer Arbeitnehmer vom Arbeitsmarkt mit der Einführung generöser Frühverrentungsregelungen im Zuge des Rentenreformgesetzes von 1972 Richtlinien, die teilweise bis heute Bestand haben. Frühverrentungen galten als einfache Möglichkeit zur Lösung von Arbeitsmarktproblemen, da sie den Betrieben eine flexible Personalanpassung ermöglichen. Beschäftigte wie Unternehmen haben die geschaffenen Regelungen in großem Umfang angenommen und zum Teil durch eigenständige betriebliche Angebote ergänzt (Deutsche Rentenversicherung Bund 2007).

Mit den Rentenreformgesetzen von 1992 und 1999 erfolgte ein rentenpolitischer Paradigmenwechsel. Man ging dazu über, die eingeführten Frühverrentungsregelungen schrittweise abzubauen, um ältere Beschäftigte länger auf dem Arbeitsmarkt zu halten. Die Altersgrenzen wurden stufenweise angehoben und deutliche Rentenabschläge bei Frühverrentung eingeführt. Desgleichen wurde der Zugang zur Altersrente wegen Arbeitslosigkeit oder nach Altersteilzeit auf die Geburtsjahrgänge vor 1952 begrenzt. Ihren aktuellen Höhepunkt fand die neue Rentenpolitik 2007 mit der Heraufsetzung der Regelaltersgrenze auf 67 Jahre. Auch die Altersgrenze für die Rente wegen Schwerbehinderung oder Erwerbsminderung wurde heraufgesetzt und die Altersrente für langjährig Versicherte auf 45 Pflichtbeitragszeiten festgesetzt. Abgeschafft wurden die Altersrente für Frauen, die Rente wegen Arbeitslosigkeit oder nach Altersteilzeit.

Neben diesen, den Übergang in den Ruhestand verzögernden Maßnahmen haben jedoch gleichzeitig noch immer arbeitsmarktpolitische Instrumente Bestand, die Frühverrentungen ermöglichen und die die genannten Regelungen teilweise konterkarieren. Dazu gehört, neben geburtsjahrgangsabhängigen Übergangsregelun- gen, an erster Stelle der Fortbestand bzw. die Ausweitung der Altersteilzeit. Diese ermöglicht Arbeitnehmern mit Vollendung des 55. Lebensjahres entweder ihre Arbeitszeit zu reduzieren oder die - von den meisten favorisierte - Blockvariante zu wählen. Auch aus betrieblicher Sicht hat sich die Altersteilzeit zu einem gerne genutzten Mittel der Frühausgliederung älterer Arbeitnehmer entwickelt, die dem häufig noch immer jugendzentristisch ausgerichtetem Personalmanagement entgegenkommt (Bellmann et al. 2003).

Die Analyse der Wege in den Ruhestand bezieht sich auf die Renteneintrittsprozesse der Geburtskohorten 1937-1941 in den Jahren 1992-2006. Die Maßnahmen und Reformen der politischen Regulierung des Renteneintritts sind zumeist an das Geburtsjahr und den Zeitpunkt des Rentenübergangs gebunden. Daher gelten für die hier untersuchten Geburtskohorten teilweise unterschiedliche institutionelle Vorgaben. Die schrittweise Anhebung der Regelaltersgrenze und die erschwerten $\mathrm{Zu}$ gangsbedingungen in sogenannte „besondere Altersrenten" ${ }^{\text {a }}$ ab 1997 betrifft insbesondere die jüngeren der hier untersuchten Geburtsjahrgänge (Schroeder 2006).

\section{3 \\ Erwerbsbeteiligung und Erwerbsausstiege Âlterer}

Ungeachtet des rentenpolitischen Paradigmenwechsels im Hinblick auf eine Verlängerung der Lebensarbeitszeit hält der Trend zu Frühverrentungen auf dem Arbeitsmarkt weiterhin an. Sind von den 55-Jährigen noch gut $70 \%$ erwerbstätig, sinkt diese Quote mit jedem Altersjahr, sodass ab dem 60. Lebensjahr weniger als die Hälfte, ab dem 62. noch ein Drittel und mit 64 Jahren weniger als $20 \%$ erwerbstätig sind (Puch 2009). Diese Entwicklung gilt gleichermaßen für Männer wie für Frauen, wobei die Erwerbsquote von Frauen noch deutlich darunter liegt. In Ostdeutschland ist der Anteil der Erwerbstätigen bei den über 55-Jährigen ebenfalls unterdurchschnittlich und wird zudem begleitet von einer hohen Arbeitslosenquote. Insbesondere in den Jahren nach der Wiedervereinigung ist die Zahl der Frühverrentungen deutlich gestiegen (BMFSFJ 2005).

In den letzten Jahren ist es zu einem Anstieg der Erwerbsbeteiligung Älterer, insbesondere von Personen zwischen 50 und 60 Jahren, gekommen (Arlt et al. 2009; Krause et al. 2008). Dieser Anstieg ist sozialpolitisch u.a. ein Effekt der Anhebung der Altersgrenzen für einen abschlagsfreien Rentenbeginn (Brussig et al. 2008). Insbesondere bei Frauen zwischen 55 und 59 Jahren ist im Zuge der allgemein gestiegenen Frauenerwerbsbeteiligung ein deutlicher Anstieg zu verzeichnen. Weitere Erklärungsfaktoren für den Wiederanstieg der Erwerbsbeteiligung Älterer ab Mitte der 1990er Jahre sind neben den geringeren Frühverrentungsmöglichkeiten das gestiegene Bildungsniveau, mit dem gemeinhin eine höhere Erwerbsbeteiligung einhergeht, eine höhere Lebenserwartung und bessere gesundheitliche Versorgung sowie der demografische Effekt des allmählichen Aufrückens der geburtenstarken Jahrgänge der 1950er Jahre in die Gruppe der älteren Arbeitnehmer (Puch 2009).

Aktuell kommen weniger als ein Drittel der Renteneinsteiger unmittelbar aus Erwerbstätigkeit bzw. sozialversicherungspflichtiger Beschäftigung. Zunehmend sind Ältere im Jahr vor dem Rentenbeginn in Altersteilzeit als Selbstständige aktiv versichert oder z. B. als Arbeitslose ohne Leistungsanspruch passiv versichert (Büttner et al. 2005). Das zentrale Merkmal der Arbeitsmarktpartizipation in den Jahren vor dem Ruhestand ist jedoch Arbeitslosigkeit (Böttcher 2005). Menschen ab 55 Jahren sind indes nicht nur häufiger als jüngere Arbeitnehmer arbeitslos, sondern auch länger (BMFSFJ 2005; Bäcker 2006). Dementsprechend stellen Ältere die größte Gruppe der Langzeitarbeitslosen mit einer Dauer von mindestens zwei Jahren. Zugenommen hat in den letzten Jahren insbesondere Arbeitslosigkeit in der Altersgruppe von 50-55 Jahren. Hinzu kommt, dass Arbeitslose ab 58 Jahren aus der Arbeitslosenstatistik herausfallen, da sie nach $\S 428$ SGB (Sozialgesetzbuch) III dem Arbeitsmarkt nicht länger zur Verfügung stehen müssen. Nicht zu vernachlässigen ist in dieser Altersgruppe zudem verdeckte Arbeitslosigkeit, wovon ältere Frauen, die sich nach vergeblicher Stellensuche vom Arbeitsmarkt zurückziehen, betroffen sein dürften. Denn mit der geringen Erwerbs-

Besondere Altersrenten sind z. B. die Altersrente wegen Arbeitslosigkeit oder die Altersrente für langjährig Versicherte. 
beteiligung Älterer geht einher, dass sie bei Neueinstellungen nur einen Anteil von 10 $\%$ ausmachen und über 50-Jährige eine deutlich geringere Einstellungschance haben als jüngere Arbeitsuchende (Brussig 2009).

Aus theoretischer Sicht wird der Prozess des Renteneinstiegs bzw. der Reduktion von Arbeitszeit im Alter durch Pushund Pullfaktoren beeinflusst (Kohli/Rein 1991). Das Spannungsverhältnis von Anreiz- (Pull) und Zwangsmechanismen (Push) ermöglicht es, den Renteneintritt theoretisch als multifaktoriellen Prozess zu fassen: Pullfaktoren betonen die Anreizwirkung von institutionellen Ausstiegsregelungen wie der Altersteilzeit, welche die Entscheidung zum vorzeitigen Ruhestand unter (ökonomischen) Nutzenerwägungen als vorteilhaft erscheinen lassen. Pushfaktoren fokussieren hingegen auf individuelle und strukturelle Zwänge älterer Arbeitnehmer, welche die Entscheidungsfreiheit bei der Wahl des Beginns der Rente einschränken. Dazu gehören die geringe Arbeitskraftnachfrage, indirekte Diskriminierung von Älteren sowie Rationalisierungsmaßnahmen zulasten von älteren Beschäftigten aufseiten der Unternehmen. Dementsprechend geben insbesondere die Chancen auf dem Arbeitsmarkt den Ausschlag für den Übergang in den Ruhestand (Radl 2007). Aber auch gesundheitliche Einschränkungen ${ }^{2}$ oder familiale Verpflichtungen beschleunigen den Renteneintritt (Voges 2008). Somit betonen Push- im Gegensatz zu Pullfaktoren die Unfreiwilligkeit bzw. das Zwanghafte des Übergangs in den Ruhestand. Zusammen verdeutlicht das Wechselspiel von Push- und Pullfaktoren, wie gesetzliche Übergangsregelungen den individuellen Renteneintritt prägen, bestimmte Verlaufsmuster forcieren, andere hingegen ausschließen. Institutionelle Vorgaben entfalten so nicht nur eine ökonomische Anreizwirkung, sondern fungieren als normative biografische Orientierungspunkte, die die Vorstellung über den „richtigen“ Zeitpunkt des Rentenbeginns prägen (Leisering 2004; Kohli 2000). Infolgedessen ist der vorzeitige Ausstieg aus dem Erwerbsleben zum gesellschaftlich akzeptierten Normalfall geworden und die Erwerbsbeteiligung bis zum 65 . Lebensjahr wird eher als Ausnahme registriert.

Somit erfolgt der Übergang in den Ruhestand mittlerweile häufig weder unmittelbar aus dem Erwerbsleben noch mit Erreichen der Regelaltersgrenze von 65 Jah- ren. Vielmehr überwiegen verschiedene Übergangsstationen, die zwischen der aktiven Erwerbsbeteiligung und dem endgültigen Ausscheiden aus dem Arbeitsmarkt liegen und durch institutionelle Regelungen sowie konjunkturelle Entwicklungen geprägt sind. Diese Verlaufsmuster bzw. Erwerbssequenzen beim Übergang in den Ruhestand sollen im Folgenden empirisch abgebildet werden. Viele Untersuchungen konzentrieren sich primär auf das Jahr unmittelbar vor dem Rentenbeginn und können so lediglich zeigen, welchen Erwerbsstatus Renteneinsteiger im letzten Jahr bzw. Monat vor dem ersten Rentenbezug innehatten (Hoffmann 2007). Dadurch bleiben jedoch Veränderungen im Erwerbsverhalten, die bereits früher eingesetzt haben, unberücksichtigt.

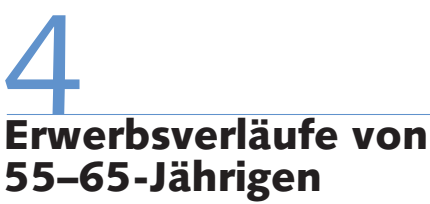

\subsection{DATENBASIS, INDIKATOREN UND METHODEN}

Die folgenden Auswertungen beruhen auf den Daten des Sozio-oekonomischen Panels (SOEP) (Wagner et al. 2008). Mit dieser Längsschnitterhebung ist es möglich, aus einer Verlaufsperspektive vollständige Erwerbsbiografien beim Übergang in den Ruhestand von ca. 800 Personen repräsentativ abzubilden. Der Untersuchungszeitraum umfasst die Jahre 1992 bis 2006, entsprechend die Geburtsjahrgänge von 1937 bis 1941. Die Angaben beziehen sich auf Personen im Alter von 55 bis 65 Jahren, die in Privathaushalten leben und für die im SOEP zu allen elf Erhebungsjahren vollständige Erwerbsinformationen vorliegen (balanced panel design). ${ }^{3}$

Zur Abbildung der Erwerbsverläufe werden die jährlichen Erwerbsstatus aller Befragungspersonen in sieben Kategorien differenziert: 1.) Vollzeiterwerbstätigkeit (VZ), 2.) Teilzeitbeschäftigung (TZ), 3.) Selbstständigkeit (SEL), 4.) Sonstige Erwerbsformen (SEW), ${ }^{4}$ 5.) Arbeitslosigkeit (ALO), 6.) Stille Reserve (RES) und 7.) Nichterwerbstätigkeit (NEW). Die verwendeten Einkommen beziehen sich jeweils auf das Vorjahr und sind preisbereinigt (zu Preisen von 2005). Haushaltseinkommen werden, um diese nach unterschiedlicher Haushaltsgröße und -struktur vergleichbar zu machen, äquivalenzgewichtet (nach der revidierten OECD-Skala).

Die Abbildung der Erwerbsverläufe erfolgt mittels einer Sequenzmusteranalyse (Brzinsky-Fay et al. 2006). Dieses methodisch innovative Verfahren erlaubt es, typische Muster, die sich in den empirischen Renteneinstiegsprozessen ergeben, anschaulich abzubilden. Denn mit der Sequenzmusteranalyse lässt sich der gesamte Erwerbsverlauf bis zum Renteneinstieg vollständig erfassen. Dabei geht es weniger um die Untersuchung von Determinanten, die zum Übergang in den Ruhestand führen, als vielmehr um die Aufdeckung von typischen (und atypischen) Erwerbsverläufen bis zum Erreichen des Ruhestandsalters. Denn ein entscheidender Vorteil dieser Methode ist, dass auch Verlaufsmuster entdeckt werden können, über die keine Vorannahmen existieren. Im Unterschied $\mathrm{zu}$ ereignisanalytischen Verfahren bleibt der Erwerbsverlauf als Ganzes im Blick. Dem liegt die theoretische wie methodische Idee zugrunde, dass nicht isolierte Ereignisse oder einzelne Determinanten den Rentenbeginn ausmachen, sondern vielmehr die komplexe Abfolge von Erwerbsstatus als Ganzes betrachtet werden muss, da auch länger zurückliegende Ereignisse - quasi als erwerbsbiografisches Gedächtnis - den Übergang beeinflussen können (MacIndoe/Abbott 2004; Aisenbrey 2000; Windzio 2001). Im Anschluss an die Sequenzmusteranalyse werden die Verläufe mittels einer Clusteranalyse gruppiert.

2 Im Falle von gesundheitlichen Einschränkungen können sozialpolitisch institutionalisierte Frühverrentungsmöglichkeiten in Anspruch genommen werden. Inwiefern es in den letzten Jahrzehnten im Zuge der steigenden Lebenserwartung einerseits sowie der erhöhten Anforderungen im Berufsleben andererseits zu einer Zu- oder Abnahme gesundheitlicher Einschränkungen am Ende des Erwerbsverlaufs gekommen ist, bleibt derzeit offen.

3 Die Analysepopulation umfasst zudem nur Personen, die zu allen Zeitpunkten im Untersuchungszeitraum vollständig beobachtet wurden. Personen, die in diesem Zeitraum gestorben, zu- oder weggezogen sind, gehören nicht zur Untersuchungspopulation, auf die sich die gewichteten Angaben der repräsentativen Bevölkerungsanteile (Verlaufstyp, Cluster) beziehen.

4 Bei den sonstigen Erwerbsformen handelt es sich in erster Linie um Altersteilzeit mit Arbeitzeit null. Diese Erwerbskategorie wird im SOEP seit 2002 erfasst. 


\subsection{ERWERBSVERLÄUFE VON 55-65-JÄHRIGEN VOM VORRUHE- STAND BIS ZUM RENTENEINTRITT}

Abbildung 1 zeigt die vollständigen Erwerbsverläufe vom 55. bis zum 65 . Lebensjahr von 787 Personen. Auf der horizontalen $\mathrm{x}$-Achse ist das Alter abgetragen, die vertikale y-Achse zeigt die einzelnen Personen. Die Erwerbsverläufe sind als horizontale Linien übereinanderliegend dargestellt, die verschiedenen Erwerbsformen sind in unterschiedlichen Grau- und Blaustufen schraffiert. ${ }^{5}$

Auf den ersten Blick sichtbar wird die Gleichzeitigkeit von Stabilität und Dynamik der Erwerbsverläufe. Denn obgleich sich die meisten Sequenzen aus den Erwerbstypen Vollzeit, Teilzeit und Nichterwerbstätigkeit zusammensetzen, variiert die Länge der Erwerbstätigkeit und damit der Zeitpunkt, zu dem der Renteneinstieg erfolgt. Zudem sind zwar einerseits $60 \%$ zwischen dem 55. und 65. Lebensjahr mindestens einmalig abhängig vollzeitbeschäftigt, andererseits kaum jemand bis zum Erreichen des 65. Lebensjahres. Vielmehr wechselt ein Großteil bereits vorher in die Nichterwerbstätigkeit. Aber auch dieser Übergang erfolgt wiederum nur für die eine Hälfte direkt, für die andere Hälfte vermittelt über eine mehr oder weniger lange Phase der Arbeitslosigkeit. Insgesamt sind $42 \%$ in den Jahren vor dem Ruhestand mindestens einmal arbeitslos, die Hälfe davon länger als zwei Jahre. Nicht überraschend ist, dass kaum jemand dieser Altersgruppe aus Arbeitslosigkeit zurück in Erwerbsarbeit wechselt, sondern überwiegend in die Nichterwerbstätigkeit, also in den Ruhestand übergeht.

Schon anhand dieser ersten Zusammenschau wird sichtbar, dass die Erwerbsverläufe dieser Altersgruppe äußerst dynamisch und heterogen sind. Im Schnitt setzten sich die Erwerbsverläufe vom 55. Lebensjahr bis zum Erreichen der gesetzlichen Altersgrenze aus drei verschiedenen Erwerbsepisoden, d.h. zwei Übergänge von einer Erwerbsform in eine andere, zusammen. Dementsprechend ist die Dauer einer Episode nur kurz. Die wenigsten Älteren können folglich auf eine kontinuierliche und dauerhafte Erwerbsbiografie bis zum Erreichen des regulären Renteneinstiegsalters zurückblicken. Ein Großteil geht vorzeitig in den Ruhestand oder zeigt wechselnde bzw. unterbrochene Erwerbsverläufe. Dadurch entsteht das Bild einer

\section{Abb. 1: Wege in den Ruhestand in Deutschland 1992-2006}

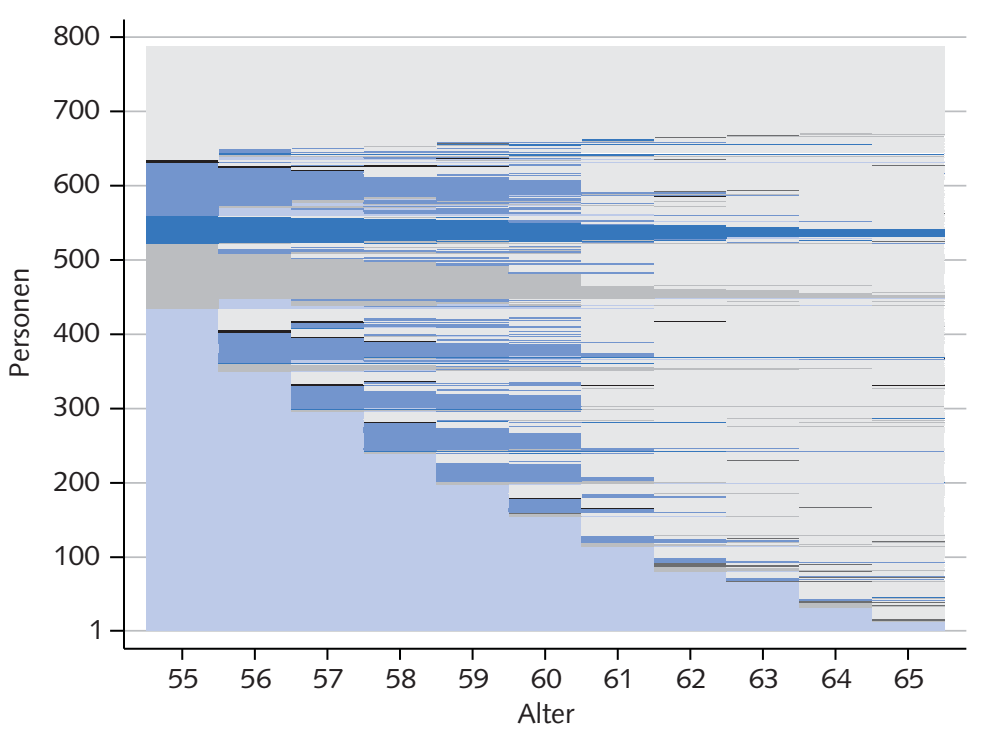

\begin{tabular}{|ll|}
\hline Vollzeiterwerbstätigkeit & Arbeitslosigkeit \\
Teilzeiterwerbstätigkeit & Stille Reserve \\
Selbstständigkeit & Nichterwerbstätigkeit \\
Sonstige Erwerbsformen & \\
\hline
\end{tabular}

stufenweise abnehmenden Erwerbsbeteiligung Älterer.

\subsection{TYPEN VON ERWERBSVERLÄUFEN BEI 55-65-JÄHRIGEN}

Noch deutlicher tritt dies zutage, wenn man die Verläufe anhand einer Clusteranalyse gruppiert. Empirisch ergeben sich daraus sechs deutlich voneinander zu unterscheidende Verlaufstypen (Abbildung 2):

Das erste Cluster Normalarbeitsverhältnis zeichnet sich durch die ununterbrochene Beschäftigung in abhängiger Vollzeiterwerbstätigkeit aus. Erst ab einem Alter von 62 Jahren findet ein langsamer Abbau der Erwerbstätigkeit statt. Der Übergang in die Nichterwerbstätigkeit erfolgt überwiegend direkt, d.h. ohne vorgelagerte Arbeitslosigkeit oder zeitliche Einschränkung in Teilzeitarbeit. Insgesamt befinden sich $12 \%$ der 55- bis 65-Jährigen in diesem Cluster. Im Kontrast dazu steht der zweite Verlaufstyp, die Frührentner aus Normalarbeit. Dieser Weg in den Ruhestand ist durch einen vorgezogenen Renteneinstieg (ebenfalls aus Vollzeitbeschäftigung) im Alter von circa 60 Jahren charakterisiert, der aus der Wahrnehmung betrieblicher oder staatlicher Frühverrentungsregelungen resultieren dürfte. Denn ein weiterer Gegensatz zum ersten Cluster ist, dass der Renteneinstieg meist nicht direkt erfolgt, sondern Phasen von Arbeitslosigkeit oder (Alters)Teilzeit vorgelagert sind. Das größte und homogenste Cluster sind die dauerhaft Nichterwerbstätigen (31\%). Dieser Verlauf ist gekennzeichnet durch die kontinuierliche Nichterwerbstätigkeit bereits ab dem 55. Lebensjahr bzw. durch einen sehr frühen Übergang aus Erwerbstätigkeit in Nichterwerbstätigkeit vor dem 60. Lebensjahr. Auf ein weiteres Viertel der 55- bis 65-Jährigen trifft der Renteneinstieg aus Arbeitslosigkeit zu. Ähnlich dem zweiten Cluster findet der Übergang in die Nichterwerbstätigkeit im Alter von 60 Jahren statt, allerdings gehen in diesem Fall sehr lange Phasen von Arbeitslosigkeit (zwischen zwei und zehn Jahren) voran. Wiederum sehr heterogen ist das fünfte Cluster, der Renteneinstieg aus Teilzeit. Gemeinsam sind den Mitgliedern dieses Ruhestandspfades relativ lange Phasen in Teilzeitbeschäftigung (im Mittel sechs Jahre) vor dem letztendlichen Ren-

\footnotetext{
5 Eine mehrfarbige Version ist auf Anfrage von den
} Autoren erhältlich. 


\section{Abb. 2: Verlaufstypen in den Ruhestand}
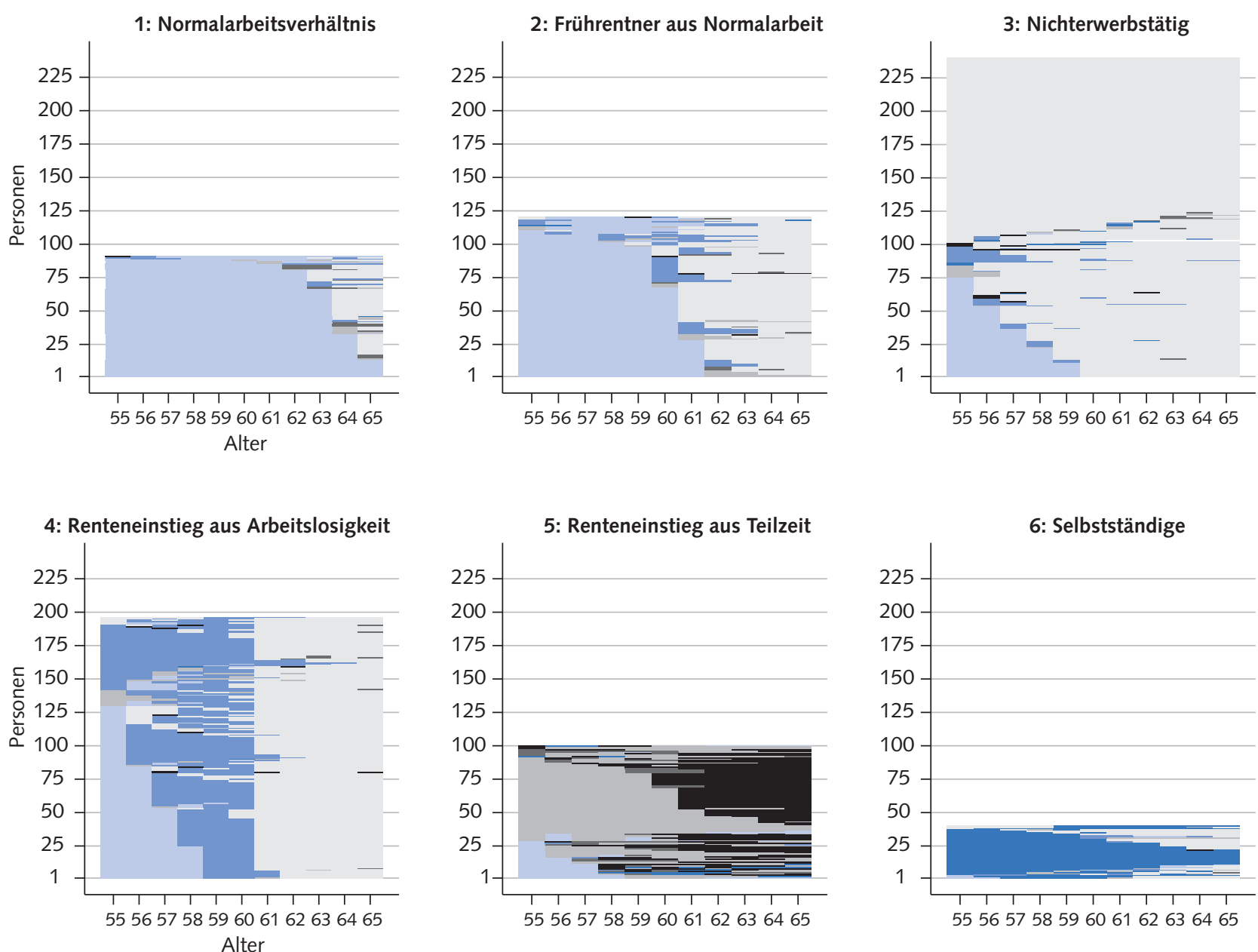

Vollzeiterwerbstätigkeit

Teilzeiterwerbstätigkeit

Selbstständigkeit

Sonstige Erwerbsformen

\section{Arbeitslosigkeit \\ Stille Reserve \\ Nichterwerbstätigkeit}

tenbeginn. Unterschiede gibt es jedoch hinsichtlich der Lage der Teilzeitarbeit im Lebensverlauf. Denn die eine Hälfte dieses Clusters ist mit 55 Jahren noch vollzeitbeschäftigt und dann kurzzeitig teilzeitbeschäftigt im Wechsel mit Arbeitslosigkeit, Nichterwerbstätigkeit oder Selbstständigkeit. Für die andere Hälfte liegt die Teilzeitbeschäftigung bereits zu Beginn der Untersuchungsdekade und wird schrittweise zugunsten von Nichterwerbstätigkeit abgebaut.

Mit $4 \%$ der Bevölkerung das kleinste Cluster bilden die Selbstständigen. Etwa ein Viertel der Selbstständigen ist bis hinein in das 65. Lebensjahr aktiv erwerbstätig, für den Großteil lassen sich mit jedem Alters- jahr schrittweise Abschiede aus der Selbstständigkeit beobachten - entweder direkt oder vermittelt über kurze Phasen in abhängiger Beschäftigung.

\subsection{ERWERBSVERLÄUFE VON 55-65-JÄHRIGEN IN SOZIO-DEMOGRAFISCHER DIFFERENZIERUNG}

Vergleicht man die Erwerbsverläufe in West- und Ostdeutschland fällt zunächst auf, dass das Normalarbeitsverhältnis primär auf Männer im Westen zutrifft ( $\mathrm{Ta}$ belle 1). Auch wenn westdeutsche Männer ebenfalls nur zu knapp einem Viertel bis zum Erreichen des Regelrentenalters in ab- hängiger Vollzeiterwerbstätigkeit beschäftigt sind, trifft dieser sogenannte Normalerwerbsverlauf weder auf Frauen noch auf ostdeutsche Männer in vergleichbarem Umfang zu. In den neuen Bundesländern dominiert demgegenüber der Renteneinstieg aus (Langzeit-)Arbeitslosigkeit. Dieser Verlauf trifft im Osten auf fast $40 \%$ der hier untersuchten Geburtskohorten zu. Allerdings muss berücksichtigt werden, dass auch Männer im Westen mit $29 \%$ häufiger in diesem Cluster sind als im erstgenannten Normalarbeitsverlauf.

Der Unterschied im Erwerbsverhalten von Frauen in West- und Ostdeutschland liegt in der hohen Nichterwerbstätigkeit westdeutscher Frauen. Fast die Hälfte der 
Tabelle 1: Verlaufstypen in den Ruhestand von Frauen und Männern in Gesamt-, West- und Ostdeutschland - in \% -

\begin{tabular}{|c|c|c|c|c|c|c|c|c|c|}
\hline & \multicolumn{3}{|c|}{ Gesamt } & \multicolumn{3}{|c|}{ Frauen } & \multicolumn{3}{|c|}{ Männer } \\
\hline & Alle & West & Ost & Alle & West & Ost & Alle & West & Ost \\
\hline Normalarbeitsverhältnis & 12,1 & 13,5 & $(7,0)$ & $(2,6)$ & $(2,5)$ & $(3,2)$ & 20,4 & 23,5 & $(9,8)$ \\
\hline Frührentner aus Normalarbeit & 13,6 & 13,2 & 15,0 & 11,6 & $(10,8)$ & $(14,9)$ & 15,3 & 15,4 & $(15,1)$ \\
\hline Nichterwerbstätige & 30,8 & 32,2 & 25,6 & 39,7 & 43,1 & 25,8 & 23,0 & 22,3 & $(25,5)$ \\
\hline Renteneinsteiger aus Arbeitslosigkeit & 26,5 & 23,0 & 39,2 & 21,3 & 16,8 & 39,7 & 31,1 & 28,7 & 38,9 \\
\hline Renteneinsteiger aus Teilzeit & 12,7 & 13,5 & $(9,7)$ & 20,5 & 21,7 & $(15,2)$ & $(5,8)$ & $(5,9)$ & $(5,5)$ \\
\hline Selbstständige & 4,4 & 4,6 & $(3,5)$ & $(4,3)$ & $(5,1)$ & $(1,2)$ & $(4,4)$ & $(4,2)$ & $(5,2)$ \\
\hline $\begin{array}{l}\text { Fallzahlen unter } 30 \text { in Klammern } \\
\text { Quelle: SOEP. }\end{array}$ & & & & & & & & $\mathrm{Ml}$ & $\mathrm{N}$ \\
\hline
\end{tabular}

Frauen in den alten Bundesländern ist bereits ab dem 55. Lebensjahr dauerhaft nichterwerbstätig, hingegen nur ein Viertel der Frauen im Osten. Auch die Frauendomäne Teilzeitarbeit kommt im Westen häufiger vor als im Osten. Ostdeutsche Frauen sind im Gegensatz dazu überproportional häufig in den Jahren vor dem Renteneinstieg arbeitslos. Damit ähnelt das Erwerbsverhalten ostdeutscher Frauen stärker dem der Männer. Wohingegen auf westdeutsche Frauen in dieser Generation in hohem Maße noch die klassische Hausfrauenehe mit dem Mann als alleinigem Ernährer und der Frau als Zuverdienerin zutrifft. Dies erklärt die hohe Stabilität und Kontinuität der Erwerbsverläufe westdeutscher Frauen. Die Erwerbsverläufe ostdeutscher Frauen sind im Vergleich dazu deutlich vielfältiger und dynamischer sowie stärker durch Erwerbsarbeit oder Arbeitslosigkeit geprägt.

Zusammenfassend lässt sich im OstWest-Vergleich konstatieren, dass die Wege in den Ruhestand im Osten brüchiger und vielfältiger sind als im Westen. Dies zeigt sich insbesondere in der hohen Betroffenheit von Arbeitslosigkeit, die sich prägend auf den Ruhestandspfad in den neuen Bundesländern auswirkt. Hingegen resultiert die Homogenität und Stabilität der Erwerbsverläufe im Westen zum einen aus dem höheren Anteil an Normalarbeitsverhältnissen von Männern und zum anderen aus der kontinuierlichen Nichterwerbstätigkeit von Frauen. Für die Renteneinsteiger in West- wie Ostdeutschland gilt jedoch, dass die aktive Beteiligung am Arbeitsmarkt äußerst gering ist und im Vergleich mit den bisherigen Querschnittsergebnissen sogar niedriger ausfällt. $\mathrm{Zu}-$ dem wird deutlich, dass das Normalarbeitsverhältnis mit jedem Altersjahr an Bedeutung verliert, wogegen Renteneinstiege aus Arbeitslosigkeit zur Normalität geworden sind. Das bedeutet jedoch, dass die Hälfte der älteren Erwerbstätigen nicht bis zum regulären Rentenalter in gewünschtem Umfang am Arbeitsmarkt partizipieren kann. In Anbetracht dieser insgesamt negativen Erwerbssituation soll abschließend ein kontrastierender Blick auf die damit einhergehenden Einkommensverläufe geworfen werden, da diese das zukünftige Renteneinkommen maßgeblich mitbestimmen.

\subsection{ERWERBSVERLÄUFE UND EINKOMMENSENTWICKLUNG}

Die individuellen Erwerbsverläufe bis zum Erreichen der gesetzlichen Altersgrenze sind eng mit der jeweiligen finanziellen Lage verbunden: Zum einen beeinflusst das Erwerbsmuster das aktuelle wie auch das daraus resultierende Renteneinkommen. Zum anderen korrespondieren Einkommensposition und-zusammensetzung mit dem Eintritt in einen bestimmten Übergangspfad. Dieses wechselseitige Verhältnis von Erwerbs- und Einkommensverlauf soll im Folgenden anhand der altersbezogenen mittleren Einkommensentwicklung (Medianeinkommen) getrennt nach Verlaufstypen exemplarisch verdeutlicht werden (Abbildungen 3 und 4). Um sowohl die unmittelbaren Auswirkungen von unterschiedlichen Wegen in den Ruhestand auf das individuelle Einkommen als auch die Bedeutung von Umverteilungsprozessen im Haushalt zu veranschaulichen, werden neben den individuellen Einkommen auch die äquivalenzgewichteten Haushaltsnettoeinkommen dargestellt. ${ }^{6}$

Auf Basis der individuellen Einkommen zeigen sich deutliche Unterschiede zwischen den Verlaufstypen: Während im Normalarbeitsverhältnis und von Frührentnern aus Normalarbeit ein hohes bzw. mittleres Einkommensniveau realisiert wird, liegen in den übrigen Verlaufstypen vergleichs- weise niedrige Einkommenspositionen vor. In den beiden erstgenannten Gruppen zeigt sich zudem deutlich ein Effekt des Übergangs in den Ruhestand, der einen Einkommensabstieg - jedoch weiterhin auf vergleichsweise hohem Einkommensniveau - mit sich führt. Bei den Nichterwerbstätigen und Rentnern aus Arbeitslosigkeit verbleiben die Einkommen auf niedrigem Niveau.

Beim Vergleich von Haushalts- und Individualebene zeigen sich besonders beim Verlaufstyp Renteneinsteiger aus Teilzeit deutliche Umverteilungsprozesse. Hier liegt der Einkommensmedian auf Haushaltsebene wesentlich höher als auf Individualebene. Die niedrige Einkommensposition der Verlaufstypen Nichterwerbstätige und Rentner aus Arbeitslosigkeit können auch im Haushaltsverbund nicht verbessert werden. Vielmehr kommt es hier zu einer Verschlechterung der mittleren Einkommensposition, d.h. zu einer Kumulierung der Problemlagen im Haushaltskontext.

Da anhand der Erwerbsverläufe Normalarbeitsverhältnis und Renteneinstieg aus Arbeitslosigkeit sowohl die unmittelbaren finanziellen Auswirkungen von unterschiedlichen Wegen in den Ruhestand als auch die Bedeutung von Umverteilungsprozessen im Haushalt besonders anschaulich werden, sollen im Folgenden für diese beiden Erwerbstypen die korrespondierenden Einkommensverläufe im Detail skizziert werden. Dies geschieht wiederum sowohl auf Ebene der individuellen Einkommen als auch auf Ebene der Haushaltseinkommen. Die Einkommen wurden dafür in Quintile eingeteilt, also in fünf gleich große Bevölkerungsgruppen. Im ersten

\footnotetext{
6 Aufgrund der teilweise geringen Fallzahlen in den Verlaufsclustern sind die dargestellten Einkommensmediane als Näherungswerte zu verstehen. Das Verlaufscluster Selbstständige umfasst lediglich 40 Beobachtungen und wird daher in den Grafiken nicht ausgewiesen.
} 
Quintil sind die ärmsten $20 \%$ der Bevölkerung, im fünften Quintil die reichsten $20 \% .^{7}$

Betrachtet man zunächst den Verlauf der individuellen Einkommen des Normalarbeitsverhältnisses (Abbildung 5), zeigen sich relativ stabile Verläufe im obersten Einkommensbereich sowie Einkommensverbesserungen, d.h. Übergänge aus dem vierten in das fünfte Quintil. Vollzeitbeschäftigte mit regulärem Rentenübertritt befinden sich also meist kontinuierlich in den oberen Einkommensklassen. Zwar sind im Haushaltsverbund die Einkommensverläufe deutlich heterogener, da offensichtlich die hohen individuellen Einkommen auf mehrere Personen umverteilt werden müssen, allerdings überwiegen noch immer Verläufe im mittleren und oberen Einkommensbereich.

Kontrastiert man diesen positiven Einkommensverlauf des Normalarbeitsverhältnisses mit dem Einkommensverlauf der Renteneinsteiger aus Arbeitslosigkeit (Abbildung 6) fällt zunächst auf, dass bereits auf der individuellen Ebene weitaus vielfältigere und instabilere Einkommensentwicklungen überwiegen, die die Brüchigkeit des korrespondierenden Erwerbsverlaufs widerspiegeln. Die Inkonsistenz dieser Erwerbsbiografie ist folglich verbunden mit einer hohen Einkommensdynamik und überwiegend absteigenden Einkommensverläufen. Entscheidend ist zudem, dass dieser negative Einkommensverlauf auch im Haushaltsverbund nicht verbessert werden kann. Hier ist wiederum erkennbar, dass es zu einer Kumulierung der Problemlagen im Haushaltskontext kommt. Ältere, die auf individueller Ebene durchaus noch mittlere Einkommenslagen erreichen, rutschen durch die Umverteilung im Haushalt in untere Einkommensklassen ab, da das Einkommen mit weiteren Personen geteilt werden muss, die häufig ebenfalls nur über ein geringes oder kein eigenes Einkommen verfügen.

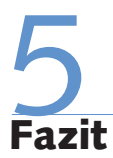

Nachdem in den 1970er und 1980er Jahren älteren Arbeitnehmern vielfältige Möglichkeiten geboten wurden, bereits vor dem 65 .

7 Die Berechnung der Quintile erfolgt jeweils auf Basis der Gesamtbevölkerung.

Abb. 3: Median des individuellen Einkommens nach Verlaufstyp - Jahresangaben in Euro -

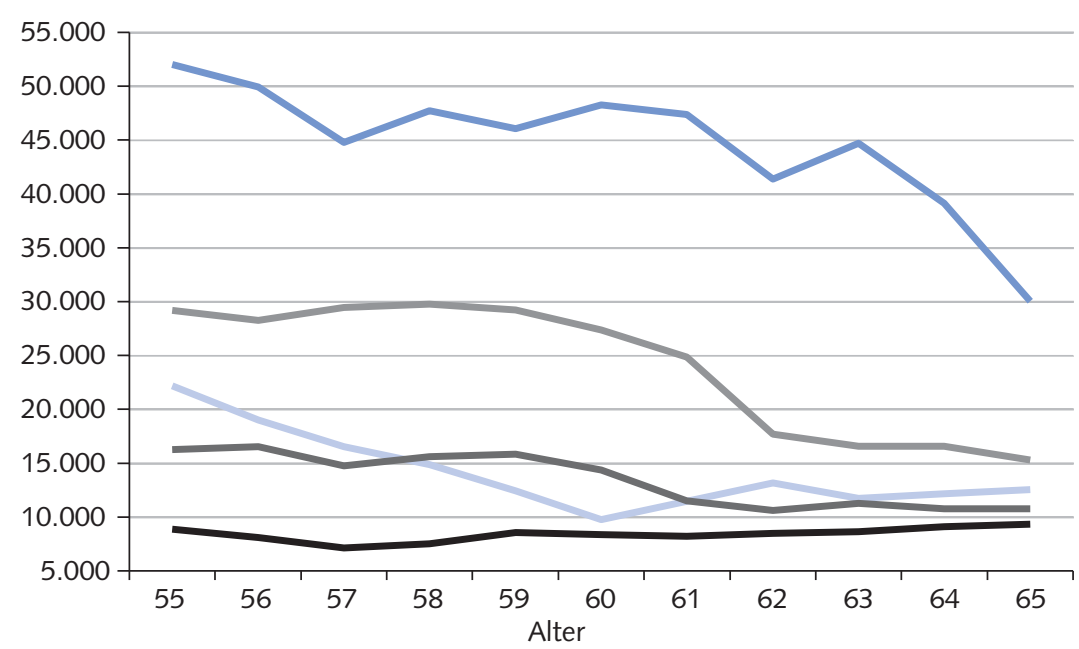

$$
\begin{aligned}
& \text { Normalarbeitsverhältnis }(\mathrm{N}=91) \\
& \text { Frührentner aus Normalarbeit }(\mathrm{N}=120) \\
& \text { Nichterwerbstätige }(\mathrm{N}=240) \\
& \text { Renteneinsteiger aus Arbeitslosigkeit }(\mathrm{N}=196) \\
& \text { Renteneinsteiger aus Teilzeit }(\mathrm{N}=100)
\end{aligned}
$$

Quelle: Daten SOEP. Gewichtete Vorjahreseinkommen, preisbereinigt zum Basisjahr 2005; Darstellung der Autoren.

WSI MitTEILUNGeN



Quelle: Daten SOEP. Gewichtete Vorjahreseinkommen, preisbereinigt zum Basisjahr 2005; Darstellung der Autoren.

WSI MITTEILUNGEN

Lebensjahr in den Ruhestand zu gehen, hat in den 1990er Jahren ein sozial- und arbeitsmarktpolitischer Wandel eingesetzt.
Das Erwerbsverhalten und die Erwerbsbeteiligung älterer Menschen sind im Zuge der Diskussion um den demografischen 


\section{Abb. 5: Einkommensverläufe des Normalarbeitsverhältnisses}

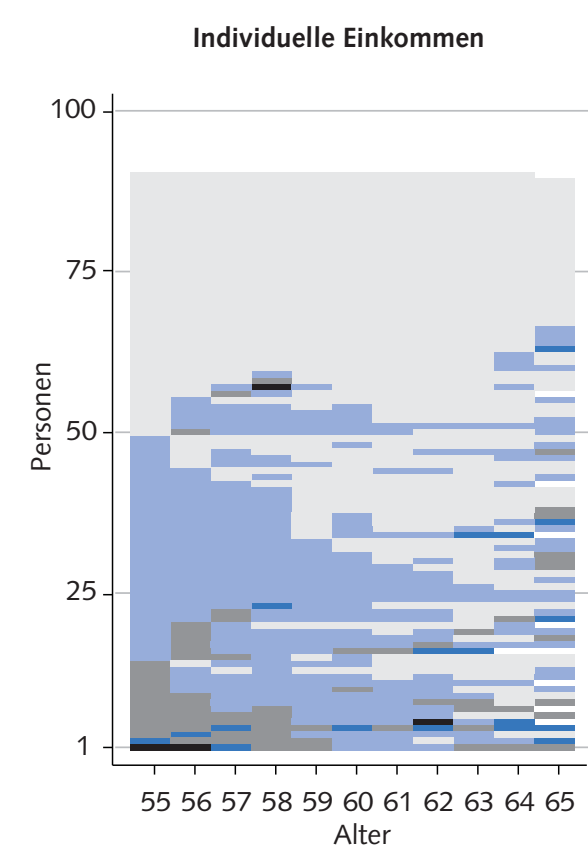

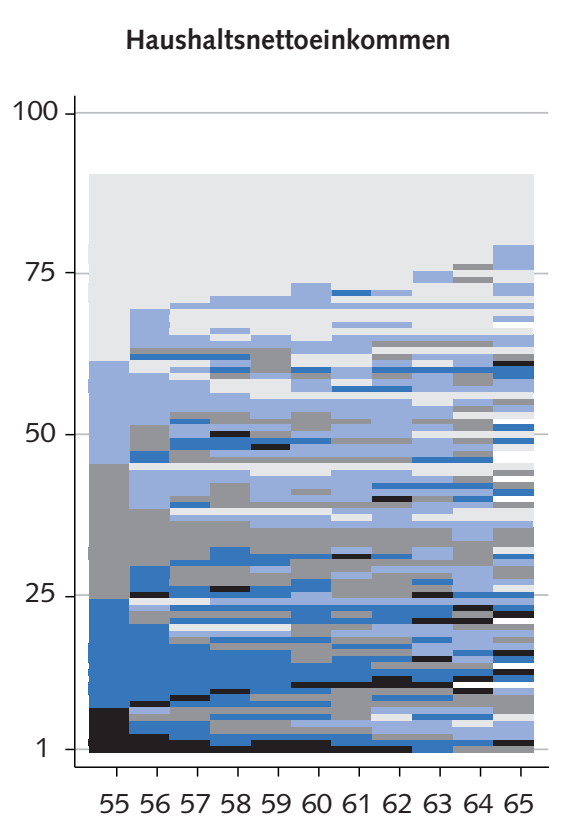

5556575859606162636465

1.EK-Quint. 2.EK-Quint. 3.EK-Quint. 4.EK-Quint. 5.EK-Quint.

Quelle: Daten SOEP; Darstellung der Autoren.

WSI MITTELUNGEN

\section{Abb. 6: Einkommensverläufe der Renteneinsteiger aus Arbeitslosigkeit}
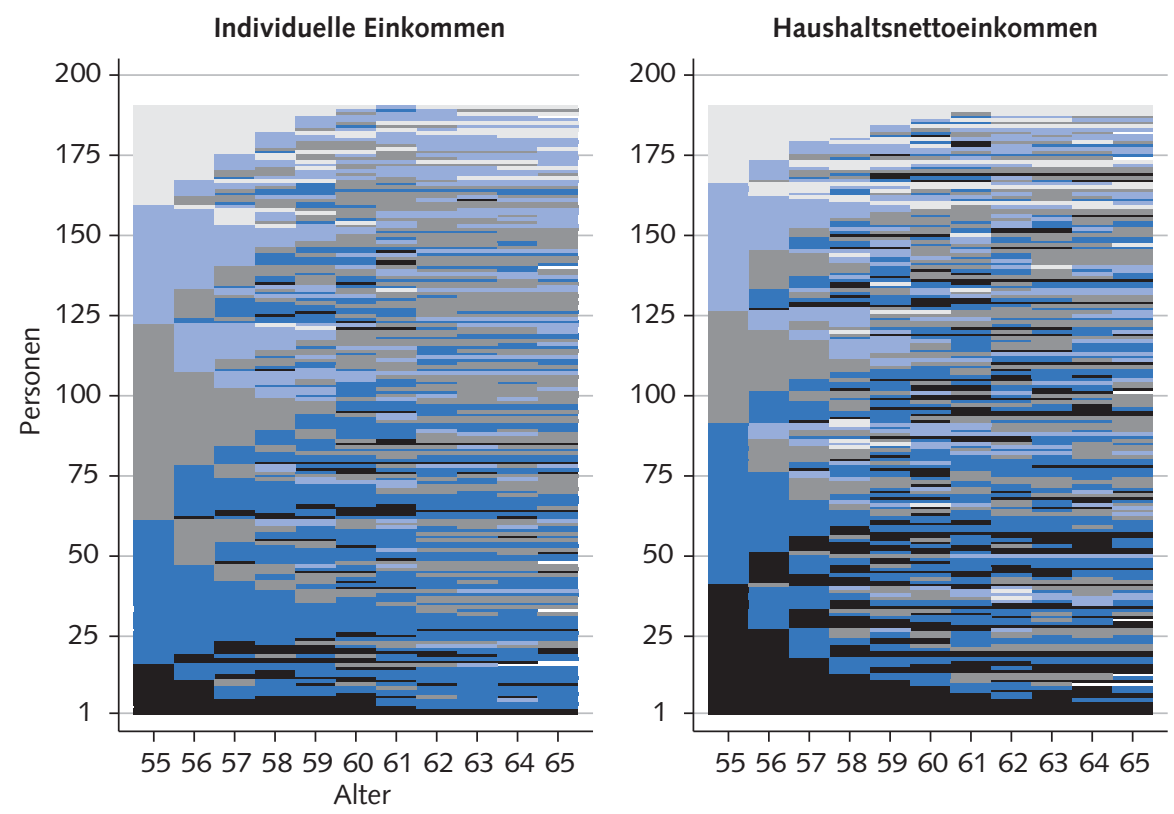

1.EK-Quint. 2.EK-Quint. 3.EK-Quint. 4.EK-Quint. 5.EK-Quint.

Quelle: Daten SOEP; Darstellung der Autoren

WSI MITTELUUNGEN

Wandel, die Alterung der Gesellschaft und den gleichzeitig prognostizierten Rückgang an Arbeitskräften in das Interesse der Öf-

ten, hat dies zur Einführung der „Rente mit 67 “ geführt. Umstritten ist allerdings, wie und ob das politische Ziel einer Erhöhung der Erwerbsbeteiligung der Bevölkerung ab 55 Jahren erfolgreich umgesetzt werden kann (Bäcker 2006). Insgesamt hat die rentenpolitische Entwicklung der letzten Jahrzehnte zu widersprüchlichen Regelungen in der aktuellen Rentenpolitik geführt. Auf der einen Seite haben Regelungen, die den vorzeitigen Renteneinstieg forcieren, weiterhin Bestand. Auf der anderen Seite wurden zahlreiche Frühverrentungsmöglichkeiten abgeschafft, der Zugang zu einem vorzeitigen Rentenbeginn verschärft und die Regelaltersgrenze angehoben.

Die vorgelegten empirischen Analysen belegen, dass die Erwerbsverläufe in den zehn Jahren vor dem regulären Renteneinstiegsalter von 65 Jahren heterogen und dynamisch sind. Dies zeigt sich am kontinuierlich abnehmenden Anteil an Normalarbeitsverhältnissen, hoher (Langzeit-)Arbeitslosigkeit, häufigen Wechseln zwischen verschiedenen Arbeitsmarktzuständen und vorgezogenen Renteneinstiegen. Dabei unterscheiden sich die Wege in den Ruhestand sowohl im Ost-West-Vergleich als auch zwischen Frauen und Männern deutlich voneinander. Darüber hinaus spiegeln die Erwerbsverläufe recht eindeutig die institutionellen Regelungen zum Renteneinstieg wieder. Zum einen gibt es die kleine Gruppe der im Normalarbeitsverhältnis Beschäftigen, die bis zum regulären Rentenalter in Vollzeit erwerbstätig sind. Zum anderen gibt es die Gruppe der vorzeitigen Renteneinsteiger aus Normalarbeit, die im Alter von 60 Jahren in den Vorruhestand gehen, teilweise flankiert von einer kurzen Phase in Altersteilzeit. Hier zeigt sich die Anreizwirkung institutioneller Ausstiegsregelungen, die als Pullfaktoren ältere Beschäftigte frühzeitig aus dem Erwerbsleben ziehen. Anhand des Verlaufs der Renteneinsteiger aus Arbeitslosigkeit wird sichtbar, dass im Anschluss an die Arbeitslosigkeit überwiegend die vorgezogene Altersrente wegen Arbeitslosigkeit in Anspruch genommen wird und ein Wiedereinstieg in die aktive Erwerbstätigkeit damit nicht gelingt. Dies verdeutlicht die geringen Arbeitsmarktchancen, die als Pushfaktoren, ältere Beschäftigte vom Arbeitsmarkt drängen.

Fokus der Studie waren nicht Determinanten des Renteneinstiegs (vgl. hierzu Radl 2007; Brussig/Stegmann 2006). Vielmehr ging es darum, den Prozesscharakter 
des Renteneinstiegs herauszuarbeiten. Dem liegt die Idee zugrunde, dass der Rentenbeginn nicht nur durch einzelne individuelle oder institutionelle Faktoren beeinflusst ist, sondern der gesamte Erwerbsverlauf mit seiner differenzierten Abfolge von Erwerbsstatus in den Jahren vor dem Ruhegang betrachtet werden muss. Die vorliegenden Ergebnisse untermauern einerseits den Befund anderer Studien, dass dem Renteneinstieg häufig Arbeitslosigkeit vorangeht (Büttner et al. 2005). Gleichzeitig gehen die Ergebnisse insofern darüber hinaus, als deutlich wurde, dass der Erwerbsstatus „arbeitslos“ vor dem Rentenbeginn keineswegs einen homogenen Zustand beschreibt, sondern einer eigenen Dynamik unterliegt. Obwohl Arbeitslosigkeit im Übergang in die Rente verbreitet ist, ergeben sich im individuellen Verlauf sehr unterschiedliche Bedeutungen und Konsequenzen. Bei den hier untersuchten Geburtsjahrgängen ist zu beachten, dass die sozialpolitische Flankierung des (vorzeitigen) Renteneintritts von älteren Personen äußerst unterschiedlich ausfällt, je nachdem, zu welchem Zeitpunkt und mit welcher "Vorgeschichte“ der Eintritt in Arbeitslosigkeit oder Nichterwerbstätigkeit erfolgt. Diese Polarisierung kann sich zukünftig verschärfen, wenn flexible $\mathrm{Zu}$ gangsmöglichkeiten in den Ruhestand weiter abgebaut werden.

Die Erwerbsverläufe spiegeln sich zudem in Sequenzmustern der individuellen und haushaltsbezogenen Einkommen wieder. Obschon die Einkommen im Haushaltsverbund umverteilt werden, führt dies nicht immer zu einem positiven Ausgleich. Das Normalarbeitsverhältnis bietet auch nach der Umverteilung im Haushaltsver- bund im Schnitt ein gutes Alterseinkommen. Gleiches gilt für Frührentner aus Normalarbeit. Allerdings greifen bei den hier untersuchten Geburtskohorten 19371941 noch die generösen Frühverrentungsmöglichkeiten, mit zum Teil abschlagsfreiem Rentenzugang. Diese finanzielle Absicherung des vorgezogenen Renteneintritts durch den Staat wird für zukünftige Rentnerkohorten nicht mehr möglich sein. Einkommensrisiken bestehen insbesondere für Nichterwerbstätige und Renteneinsteiger aus Arbeitslosigkeit. Die hier untersuchten Geburtskohorten 1937-1941 profitieren zwar noch von sozialpolitischen Umverteilungsprozessen beim Übergang in die Rente. Für zukünftige Rentnerkohorten kann der Abbau der staatlichen Absicherung mit Einkommensrisiken verbunden sein, da in diesen Verlaufstypen sowohl die Anbindung an den Arbeitsmarkt als häufig auch der Ausgleich durch ein höheres Familieneinkommen fehlen. Besonders profitieren durch die Einkommensumverteilung im Haushaltsverbund derzeit die Renteneinsteiger aus Teilzeit. Hierbei handelt es sich überwiegend um Frauen, die im Haushaltskontext meist gut abgesichert sind. Allerdings entstehen auch für diese Personengruppe im Fall einer Scheidung erhebliche Einkommensrisiken.

Deutlich wurde, dass es insbesondere bei Personen mit ohnehin prekären Verlaufsmustern - Renteneinsteiger aus Arbeitslosigkeit und dauerhaft Nichterwerbstätige - zu einer Kumulation negativer Erwerbskonstellationen im Haushalt und zu insgesamt absteigenden Einkommensverläufen kommt, die Gefahr laufen, dauerhaft hinter der allgemeinen Entwicklung zurückzubleiben. Vor dem Hintergrund der langfristigen Herabsenkung des gesetzlichen Rentenniveaus und der unzureichenden Abdeckung der Erwerbsnachfrage vor Renteneintritt ergeben sich hieraus in besonderem Maße Risiken für zukünftige Rentnerkohorten. Zudem verdeutlicht sich anhand der Kontrastierung der Einkommensverläufe der verschiedenen Wege in den Ruhestand, welche Auswirkungen das Erwerbsverhalten in den Jahren vor dem Rentenbeginn auf die Einkommenslage hat. Dies war für die individuellen Einkommen nicht anders zu erwarten. Ausschlaggebend ist, dass sich negative individuelle Einkommenslagen im Haushaltskontext tendenziell sogar noch verstärken. Auch dies wirkt sich in Richtung einer Polarisierung der Rentenzugangsmuster und resultierenden Einkommen im Ruhestand aus.

Derzeitige Rentnerkohorten erreichen zwar ein mit der Gesamtbevölkerung vergleichbares Einkommensniveau (Krause et al. 2008), allerdings beruht dieses meist auf kontinuierlichen Erwerbsbiografien, wie sie im Westen Deutschlands und insbesondere in der ehemaligen DDR lange Zeit üblich waren. Die Erwerbsverläufe aktueller und zukünftiger Renteneinsteiger sind hingegen zunehmend brüchig und instabil. Dies kann zu geringen Rentenanwartschaften führen. Daher besteht ein deutlicher Forschungs- und Handlungsbedarf, um die zunehmende arbeitsmarktinduzierte Dynamik in den letzten Jahren vor dem Renteneinstieg sozialpolitisch abzusichern, prekäre Übergangsprozesse zu vermeiden und so potenziellen Polarisierungstendenzen durch Kumulationseffekte im Haushalt und künftiger Altersarmut entgegenzuwirken. 


\section{LITERATUR}

Aisenbrey, S. (2000): Optimal matching analyse, Opladen Arlt, A./Dietz, M./Walwei, U. (2009): Nicht alles ist Konjunktur - Besserung für Ältere am Arbeitsmarkt, IAB-Kurzbericht 16, Nürnberg Bäcker, G. (2006): Rente mit 67: Länger arbeiten oder länger arbeitslos? Probleme einer Heraufsetzung der Altersgrenze, in: Wirtschafts- und Sozialpolitisches Forschungs- und Beratungszentrum der Friedrich-EbertStiftung (Hrsg.): Rente mit 67. Steuerungspotenziale in der Renten- und Beschäftigungspolitik, Bonn, S. 6-23

Bellmann, L./Kistler, E.; Wahse, J. (2003): Betriebliche Sicht- und Verhaltensweisen gegenüber älteren Arbeitnehmern, in: Aus Politik und Zeitgeschichte 20, S. 26-34

Bundesministerium für Familie, Senioren, Frauen und Jugend (BMFSFJ) (2005): Fünfter Bericht zur Lage der älteren Generation in der Bundesrepublik Deutschland, Berlin

Böttcher, S. (2005): Übergangsprozesse am Ende des Erwerbslebens ein Projektbericht, in: DRV-Schriften 55, S. 226-241

Brzinsky-Fay, C./Kohler, U./Luniak, M. (2006): Sequence analysis with Stata, in: The Stata Journal 4, S. 435-460

Brussig, M. (2009): Neueinstellungen von Älteren: Keine Ausnahme, aber auch noch keine Normalität, in: Altersübergangs-Report 01, HansBöckler-Stiftung

Brussig, M./Knuth, M./Wojtkowski, S. (2008): Die Weichen sind gestellt, doch der Streckenausbau ist noch nicht abgeschlossen, in: Altersübergangs-Report 03, Hans-Böckler-Stiftung

Brussig, M./Stegmann, T. (2006): Wer geht vorzeitig in Rente? Eine Analyse mit den Individualdaten des Versichertenrentenzugangs 2004, DRV-

Schriften 55, Berlin

Büttner, R./Knuth, M./Wojtkowski, S. (2005): Die Kluft zwischen Erwerbsaustritt und Renteneintritt wird wieder größer, in: AltersübergangsReport 03, Hans-Böckler-Stiftung

Deutsche Rentenversicherung Bund (Hrsg.) (2007): Rentenversicherung in Zeitreihen, DRV-Schriften 22, Berlin

Hoffmann, H. (2007): Wege in den Ruhestand, in: Deutsche Rentenversicherung 4-5, S. 298-320

Kohli, M. (2000): Altersgrenzen als gesellschaftliches Regulativ individueller Lebenslaufgestaltung, in: Zeitschrift für Gerontologie und Geriatrie 33 (Suppl. 1), S. 15-23
Kohli, M./Rein, M. (1991): The changing balance of work and retirement, in: Kohli, M/Rein, M./Guillemard, A.-M./Gunsteren, H. van (Hrsg.): Time for retirement. Comparative studies of early exit from the labor force, Cambridge, S. 1-35

Krause, P./Möhring, K./Zähle, T. (2008): Wohlstandsdisparitäten bei Älteren in Ost- und Westdeutschland, in: Deutsche Rentenversicherung 1 , Berlin, S. 40-59

Leisering, L. (2004): Government and the life course, in: Mortimer, J. T./ Shanahan, M. J. (Hrsg.): Handbook of the life course, New York, S. 205225

MacIndoe, H./Abbott, A. (2004): Sequence analysis and optimal matching, in: Hardy, M./Bryman, A. (Hrsg.): Handbock of data analysis, London, S. 387-406

Puch, K. (2009): Erwerbsbeteiligung älterer Arbeitnehmer, in: Statistisches Bundesamt (Hrsg.): STATmagazin vom 13.1., Wiesbaden

Radl, J. (2007): Individuelle Determinanten des Renteneintrittsalters.

Eine empirische Analyse von Übergängen in den Ruhestand, in: Zeitschrift für Soziologie 1, S. 43-64

Schroeder, W. (2006): Vergangenheit, Gegenwart und Zukunft des Vorgezogenen Renteneinstiegs, in: Wirtschafts- und Sozialpolitisches Forschungs- und Beratungszentrum der Friedrich-Ebert-Stiftung (Hrsg.): Rente mit 67. Steuerungspotenziale in der Renten- und Beschäftigungspolitik, Bonn, S. 6-23

Voges, W. (2008): Soziologie des höheren Lebensalters. Ein Studienbuch zur Gerontologie, Augsburg

Wagner, G./Goebel, J./Krause, P./Pischner, R./Sieber, I. (2008): Das Sozio-oekonomische Panel (SOEP): Multidisziplinäres Haushaltspanel und Kohortenstudie für Deutschland - Eine Einführung (für neue Datennutzer) mit einem Ausblick (für erfahrene Anwender), in: AStA Wirtschafts- und Sozialstatistisches Archiv 4, S. 301-328

Windzio, M. (2001): Übergänge und Sequenzen. Der Einfluss von Arbeitslosigkeit auf den weiteren Erwerbsverlauf, in: Sackmann, R./Wingens, M. (Hrsg.): Strukturen des Lebenslaufs, Weinheim, S. 163-198 